Citius., Altius, Fortius- 2018, 11(2), pp. 9-18

\title{
UN ENFOQUE FILOSÓFICO DE LOS DESAFÍOS DE LA PROMOCIÓN DE PROGRAMAS PARA CONVERTIR A LOS ATLETAS OLÍMPICOS EN MODELOS A SEGUIR ${ }^{1}$
}

\author{
Francisco Javier López Frías \\ The Rock Ethics Institute \\ Universidad Estatal de Pensilvania(EE.UU.) \\ fj113@psu.edu
}

Fecha de recepción: julio 2018

Fecha de aceptación: octubre 2018

DOI: http://doi.org/10.15366/citius2018.11.2.002

\section{Resumen:}

En este artículo, se analizan las dificultades a la hora de desarrollar programas para la conversión de atletas olímpicos en modelos a seguir. Para ello, primero se mostrará cómo los atletas son tomados como modelos a seguir en nuestra sociedad. Posteriormente se expondrá un análisis filosófico del concepto de modelo a seguir, basado en la distinción que establece Feezell entre «modelo a seguir» y «ejemplar moral». Tras ello, se analizará si los atletas tienen la obligación de servir de modelo para otros, distinguiendo entre dos propuestas al respecto, a saber: la ejemplarista y la escéptica. Por último, serán analizados cuatro desafíos surgidos a raíz de las distinciones conceptuales.

Palabras clave: Ética, modelo a seguir, atleta, olimpismo.

Title: THE PHILOSOPHICAL THEORIES ON SPORT ROLE MODELING AND THE CHARACTERISTICS OF ROLE-MODEL PROGRAMS

\begin{abstract}
:
In this paper, I analyze the difficulties involved in developing role-model programs in Olympic sports. To do so, I will show how today's athletes are regarded as role models in the larger society. Then, drawing on Feezell's distinction between "role models" and "moral exemplars," I will provide a philosophical analysis of the concept of "role model." Next, I will explore the question whether athletes have role modeling obligations, drawing a distinction between two approaches to the issue, namely: exemplarism and scepticism. Lastly, I will examine four challenges for the development of role-model programs that arise from the philosophical distinctions presented in the paper. Keywords: ethics, role model, athlete, Olympism.
\end{abstract}

Key words: Ethics, model to follow, athlete, Olympism.

\footnotetext{
${ }^{1}$ Este texto corresponde a la versión en castellano de la ponencia del autor titulada «The philosophical theories on sport role modeling and the characteristics of role-model programs» presentada en las jornadas correspondientes a la 14 Sesion International conjunta para Presidentes y Directores de Academias Olímpicas Nacionales y miembros de Comités Olímpicos Nacionales, celebrada por la Academia Olímpica Internacional entre el 6 y el 13 de Mayo del 2018, en su sede de Olimpia (Grecia).
} 
«Una misma es la raza de los hombres, una misma la de los dioses, y de una misma madre (nacidos) alentamos unos y otros. Pero nos separa un poder todo diverso, por modo que nada es la una, mientras el cielo broncíneo permanece siempre en asiento seguro. Pero en algo, con todo, nos acercamos - sea en nuestro espíritu, sea por naturaleza - a los Inmortales, aunque ni durante el día ni en la noche sabemos nosotros hacia qué meta nos prescribió correr el Destino» (Píndaro, 1984, 242-243).

\section{El atleta: figura moral de la sociedad}

En la obra Tras la Virtud, el filósofo Alasdair MacIntyre (2013) defiende que una sociedad puede ser comprendida a través de las figuras morales que definen su tradición moral. Tales personajes son individuos que encarnan las ideas y creencias morales sobre las que se sustenta la evaluación y el juicio moral de los miembros de la sociedad. Por ejemplo, apunta MacIntyre, el guerrero era el personaje moral clave de la sociedad homérica, pues encarnaba las virtudes y valores del coraje y la lucha. Igualmente, en nuestra sociedad, el burócrata y el asceta actúan como figuras morales al personificar la racionalidad técnica y la persecución egoísta de satisfacer deseos respectivamente. De modo coloquial, el término empleado para referirse a lo que MacIntyre denomina como «caracteres morales» es «modelos a seguir», que, según el Diccionario MerriamWebster, se refiere a «individuos cuyo comportamiento en un papel determinado es imitado por otros». A menudo, los atletas son considerados como modelos a seguir en la sociedad actual. Por ejemplo, la filósofa española Adela Cortina, basándose en MacIntyre, afirma que uno de los caracteres morales clave de nuestro tiempo es el deportista (Cortina, 2012; 2014).

Teóricos del deporte como Eduardo Galeano (1995) y Jorge Valdano han reflexionado sobre el papel que el mismo juega en la sociedad y han defendido que los deportistas son modelos a seguir. Por ejemplo, este último afirmaba en una reciente entrevista que «en la sociedad actual no hay más héroes que los deportistas» (González, 2012). De hecho, la XI encuesta «Qué quiere ser de mayor» realizada por la Fundación Adecco a niños y niñas españoles de entre 4 y 16 años muestra que el $36,5 \%$ de los niños quieren tener un trabajo relacionado con el deporte, especialmente, futbolistas (26,7\%). Igualmente, en Inglaterra, una encuesta reciente realizada por el Servicio Nacional de Ciudadanos (NCS) a aproximadamente 1.000 adolescentes de 16 a 17 años, el 59\% de los participantes admitió haber imitado el comportamiento de personas famosas y presentó las siguientes razones para hacerlo: «Me alientan a querer tener éxito», «me proporcionan alguien a quien admirar», «me hacen querer ser una mejor persona» $y$ «me obligan a salir fuera de mi zona de confort».

Así, pues, seis atletas fueron incluidos en la lista de las «100 personas más influyentes de 2017» de la prestigiosa revista TIME, a saber: Simon Biles, Colin Kaepernick, Neymar, Lebron James, Tom Brady y Conor McGregor. Los columnistas de la revista justificaban la selección de estos atletas en función de su potencial para inspirar a otros. Así, por ejemplo, de la estrella del baloncesto LeBron James se dice que es «una inspiración para todos los estadounidenses, una prueba de que el talento combinado con la pasión, la tenacidad y la decencia pueden reinventar lo posible». Sobre Tom Brady, el exitoso quarterback de fútbol americano, se dice que a través de su «dieta monástica, entrenamiento intenso y una implacable e inspiradora positividad, Tom se construyó desde cero». El también jugador de fútbol americano, Colin Kaepernick se presenta como alguien que «ha demostrado coraje ejerciendo su derecho garantizado a la libertad de expresión ... a un costo personal». Respecto al futbolista brasileño Neymar, «vive para jugar el juego ... de la misma manera que lo hizo cuando era niño». Por último, la gimnasta americana Simone Biles se percibe como alguien que «transmite el siguiente mensaje a todos los que la están mirando: no importa por lo que estés pasando en la vida o cuáles sean las circunstancias, todavía puedes ser el

Citius, Altius, Fortius Volumen 11 ne 2. Noviembre, 2018. ISNN. 2340-9886.

http://doi.org/10.15366/eitius2018.11.2 
número 1. Solo tienes que trabajar duro»(The World's 100 Most Influential People, 2017). Sacrificio, creatividad, disciplina, jovialidad, coraje y perseverancia son las principales virtudes que los seis atletas encarnan y que les convierten en figuras morales de nuestro tiempo.

Muchos atletas han aceptado gratamente tener un papel influyente en la sociedad, contribuyendo a la expectativa ampliamente aceptada de que deben utilizar su influencia para modelar el buen comportamiento de aquellos que les admiran. Sin embargo, tal expectativa ha sido disputada por algunos filósofos del deporte, que han planteado dos tipos de preocupaciones. Uno tiene que ver con el tipo de obligaciones que conlleva ser un modelo que imitar, el otro tipo de preocupación es relativo a la medida en que se debe esperar y exigir a los atletas que actúen como modelos a seguir.

\section{2. ¿Qué es un modelo a seguir? ¿Qué comportamientos modelan los atletas?}

La concepción del atleta como un personaje moral clave no es exclusiva de nuestro tiempo. En muchas épocas de la humanidad, la relevancia del deportista ha transcendido el terreno de juego. El atleta siempre ha sido un ídolo secular. Por ejemplo, en la oda de Píndaro al comienzo de este artículo, se concibe al deportista como modelo a seguir porque es capaz de alcanzar la inmortalidad propia de los dioses a través del desarrollo de sus habilidades en un grado excelente. Además del atleta griego, en la historia, podemos encontrar una infinidad de atletas que sirvieron como inspiración para sus contemporáneos: el gladiador romano, el caballero medieval, el deportista amateur y el atleta profesional actual (Amidon, 2012).

En un artículo publicado en Bleacher Report, el periodista deportivo James Riggio enumera los mejores modelos a seguir en los últimos 25 años. Según él, algunos de los comportamientos que hacen que los atletas en su lista sean buenos modelos de conducta son de tipo muy diverso y variado. A saber:

«(...) demostrar una gran competitividad, no cometer faltas (durante el juego), hacer más cosas buenas que malas, ser galardonado con una prestigiosa beca académica, hacer trabajo comunitario, recaudar dinero para niños con cáncer, personificar el espíritu de lucha, ser un ciudadano modelo, participar en organizaciones benéficas y mostrar carácter, liderazgo y fe en el baloncesto» (Riggio, 2012).

Las razones anteriores proporcionadas por Riggio son muy diversas desde el punto de vista de la moral, ya que van desde aquellas de tipo amoral relativas a desempeñar correctamente un papel determinado (por ejemplo, la competitividad y la dedicación), hasta aquellas que son exigibles moralmente (hacer más cosas buenas que malas) pasando por otras que son consideradas como supererogatorias, es decir, como moralmente alabables, pero no exigibles (estar involucrado en organizaciones benéficas). Además, no hemos de olvidar que los atletas también pueden actuar como malos modelos a seguir, lo cual Riggio parece pasar por alto. Es decir, pueden modelar comportamientos negativos y contravalores en aquellos que les imitan y admiran.

Dados los diferentes tipos de comportamientos que los atletas pueden inspirar, los filósofos sostienen que la primera tarea que deben llevar a cabo los análisis filosóficos del atleta como modelo a seguir es el significado del concepto «modelo a seguir». Según el filósofo Randolph Feezell (2005), dicho término tiene dos significados. Por un lado, los modelos a seguir se conciben de forma limitada a una profesión o actividad en particular. Desde esta perspectiva, los atletas deben modelar el comportamiento de aquellos dentro del ámbito deportivo, es decir, son modelos a seguir en tanto que atletas. Por ejemplo, Simone Biles modela el comportamiento de gimnastas que quieren ser excelentes en dicha competición deportiva. En este sentido estrecho, «querer ser como 
Biles» significa actuar, entrenar y comportarse como gimnasta en tanto que gimnasta. Feezell emplea el término «modelos a seguir» para referirse a esta afección del concepto.

Por otro lado, afirma Feezell, los atletas son considerados como modelos a seguir de un modo más amplio, a saber, como individuos cuyo comportamiento se imita en todas las esferas de la vida porque ejemplifican los rasgos morales y los valores que la sociedad considera como buenos y correctos. Desde esta perspectiva, «querer ser como Biles» significa emular su estilo de vida porque ella supone un ejemplo de cómo «todo se puede lograr con trabajo duro» (Jones, 2017). Para referirse a este significado amplio del concepto «modelo a seguir», Feezell emplea el término «ejemplares morales».

En resumen, basándonos en los dos modelos principales del atleta como modelo a seguir en la disciplina de la filosofía del deporte, puede decirse que hay dos ámbitos en que los deportistas pueden modelar el comportamiento de aquellos que les imitan y admiran. Uno es el relativo al actuar como buenos o malos modelos para que otros atletas se conviertan en mejores o peores en su profesión. El otro es el que concierne a servir como ejemplares morales que encarnan y ejemplifican valores y rasgos morales buenos (o malos) en la vida cotidiana.

\title{
3. ¿Deberían los atletas ser modelos a seguir? ¿Qué obligaciones tienen?
}

\author{
Según el filósofo Earl Spurgin:
}

«[un] modelo a seguir es un individuo que tiene la obligación especial de modelar el comportamiento apropiado para una persona o grupo de personas en particular ... Los modelos a seguir están obligados a actuar de manera que los individuos o grupos puedan observar el comportamiento apropiado» (2017, p. 188).

Para él, no sólo es que los atletas modelan el comportamiento atlético y/o moral, sino que, más bien, están moralmente obligados a hacerlo. La discusión sobre la naturaleza de tal obligación moral es clave en el debate ético sobre los modelos a seguir en el deporte.

En una columna publicada en 1993 en la prestigiosa revista deportiva Sports Illustrated, el famoso jugador de la NBA, Karl Malone, manifestó su posición respecto al debate en torno a los modelos a seguir argumentando:

«No elegimos ser modelos a seguir, somos elegidos. Nuestra única posibilidad de elección es optar entre ser un buen modelo a seguir o uno malo ... Me encanta ser un modelo a seguir, y trato de serlo de modo positivo» (Malone, 1993).

No obstante, no todos los atletas están contentos con tener obligaciones como modelos a seguir y las aceptan del mismo modo que Malone. Por ejemplo, como respuesta a Malone, el también ex jugador de la NBA Charles Barkley protagonizó un anuncio de la compañía de deportes Nike, en el cual dijo:

«No soy un modelo a seguir. No me pagan por ser un modelo a seguir. Me pagan para hacer estragos en la cancha de baloncesto. Los padres son modelos a seguir. El hecho de que sea bueno al baloncesto no significa que pueda criar a sus hijos» (Newsweek, 1993).

Las posiciones de Malone y Barkley hacia el papel modelo que los deportistas deben jugar en la sociedad se reflejan en el debate filosófico en torno al deporte (Wellman, 2003). 
La posición de Charles Barkley, que se resume en su conocida frase: «No me pagan por ser un modelo a seguir, sino por causar estragos en la cancha baloncesto» ilustra el enfoque escéptico. Desde este punto de vista, los atletas no deben ser considerados ni como modelos a seguir, ni como ejemplares morales, salvo que voluntariamente elijan serlo. En este modelo, los atletas son profesionales cuyas obligaciones se restringen a su empleador y compañeros de trabajo. Es decir: las obligaciones de los atletas se limitan a mostrar un buen rendimiento deportivo, por ejemplo, deben mostrar dedicación y compromiso con su profesión. Cualquier otra obligación adicional, como las obligaciones de convertirse en ejemplares morales, debe ser elegida voluntariamente por los atletas. Desde esta perspectiva, la responsabilidad de modelar el buen comportamiento tanto en el terreno de juego como fuera de él, es una cuestión de elección subjetiva, no de obligación objetiva ligada a su profesión. Así, por ejemplo, el futbolista del Real Madrid C.F., Álvaro Arbeloa, en reacción a las críticas que se generaron respecto al mal ejemplo que se daba en cada duelo que enfrentaba a su equipo con su enemigo histórico, el F.C. Barcelona, afirmaba que:

«[a]hora se hace mucha incidencia en que tenemos que ser ejemplos para los niños. Yo no tengo que ser ejemplo para nadie. Tengo que ser ejemplo para mis hijos y punto. Nunca salgo al campo pensando en los niños. Ni pensando en hacer daño a nadie o queriendo lesionar. Soy jugador de fútbol y salgo pensando en lo mejor para mi equipo. Tuve un padre que cuando veíamos algo en la televisión me decía: 'Mira, esto no se hace. Esto está bien y esto está mal'» (Jabois, 2014).

El segundo enfoque filosófico respecto al atleta como modelo a seguir es el ejemplarismo. Éste se sustenta sobre la idea de que los atletas deben actuar como modelos a imitar, les guste o no. Las obligaciones de modelado de comportamiento están integradas en su profesión, es decir, son propias e inseparables de la misma. La afirmación de Malone de que los atletas «no eligen ser modelos a seguir, [sino que son] elegidos» ilustra este enfoque. Para el ejemplarismo, la obligación de actuar como modelo a seguir no es el resultado de una elección subjetiva, sino un elemento objetivo intrínseco a la profesión atlética. Así, pues, el ejemplarismo otorga responsabilidades adicionales al atleta, pues se le pide que, además de ser exitoso en su profesión, ejemplifique buenos valores y virtudes para otros (lo cual, como vimos en el apartado anterior, puede tener dos acepciones. Bien puede exigirse al deportista que sea bien un modelo a seguir dentro del terreno de juego o bien que se convierta en un ejemplar moral).

\section{Las limitaciones de las teorías filosóficas del atleta como modelo a seguir.}

Ambos enfoques éticos relativos al atleta como modelo a seguir, escepticismo y ejemplarismo tienen limitaciones, especialmente cuando se evalúan en función de los valores y objetivos del Olimpismo. El enfoque escéptico, por ejemplo, está limitado, al menos, de dos maneras. Primero, ciertas posiciones y profesiones sociales sí exigen que los individuos sean modelos a seguir. Por ejemplo, los padres deben modelar el comportamiento de sus hijos. Algunos argumentan que la profesión atlética también incluye tal obligación. Además, los códigos de ética profesionales y los acuerdos contractuales a menudo incluyen la obligación de comportarse de modo ejemplar para otros. Por ejemplo, los equipos deportivos profesionales y las organizaciones deportivas exigen a los atletas participar en campañas de caridad para inspirar a otros a participar en comportamientos altruistas.

Como es bien sabido, el Barón Pierre de Coubertin (2000), padre del Olimpismo moderno, concibió el Olimpismo como un intento de infundir el espíritu de superación olímpica de la antigüedad. A través de ello, no sólo pretendía reformar el comportamiento de aquellos que participaban en los deportes profesionales y olímpicos, sino también y, sobre todo, de la sociedad moderna en general. Coubertin pensaba que la recuperación del espíritu de Olimpia ayudaría a promover la búsqueda del cultivo de los aspectos humanos de las personas, contrarrestando el 
individualismo y la mentalidad de ganar a toda costa que prevalecía en los deportes y en la sociedad en su época. Olimpia, para Coubertin, simbolizaba un mundo dónde los individuos se involucraban en proyectos comunes, no para lograr objetivos egocéntricos, sino para buscar cooperativamente la excelencia, creando una comunidad donde todos y cada uno pudieran desarrollarse como personas. Los atletas olímpicos, por lo tanto, no sólo portan la antorcha olímpica de tipo material, sino también la antorcha simbólica conformada por los valores que encarna Olimpia y Los Juegos Olímpicos. Además, de un modo más general, dado el carácter social del ser humano, a todos y cada uno de los miembros de la sociedad, incluyendo los profesionales del deporte, tienen obligaciones públicas. De este modo, Javier Gomá afirma:

«[t]odos somos ejemplos para todos, que todo hombre es ejemplo para los demás y los demás lo son para él, porque el yo vive en una red de influencias mutuas a la que le es imposible hurtarse» (Gomá, 2009, sección II).

La segunda limitación del enfoque escéptico es que la ética no es un disfraz que podamos ponernos y quitarnos a nuestra voluntad (López Frías, 2014). Los seres humanos somos seres éticos por naturaleza, y también lo son nuestras acciones; ya sea porque son el resultado de una visión particular de la buena vida o porque afectan las posibilidades que otras personas poseen para lograr una vida buena. La naturaleza moral de la acción humana no puede ser puesta entre paréntesis; los individuos no pueden elegir qué tipo de obligaciones morales cumplir. La obligación moral no es una cuestión de elección subjetiva. Si los atletas tienen obligaciones morales como modelos a seguir, entonces deben cumplirlas para hacer lo correcto.

Cualquier explicación ética del atleta como modelo a seguir debe tener en cuenta el hecho de que algunas personas toman a éstos tanto como modelos a seguir, como ejemplares morales. Debido a que los atletas tienen más capacidad para afectar a los demás, sus acciones tienen implicaciones éticas mayores, lo cual les hace más responsables con respecto a aquellos afectados por sus acciones. Como afirma el filósofo Hans Jonas: «la responsabilidad surge del poder» (veáse López Frías, 2018). Por ejemplo, si los individuos de la sociedad celebran, admiran e imitan la actitud indiferente que algunos atletas tienen hacia la trampa y la vulneración de las reglas del deporte (y de la vida cotidiana en algunos casos), el engaño en la sociedad en general podría normalizarse, o incluso justificarse, a los ojos de algunos de esos individuos. Esto tendría un impacto negativo en la sociedad, del cual los atletas deberían hacerse responsables en cierto modo.

El enfoque ejemplarista tiene tres limitaciones. La primera es que esperar o exigir que los atletas se comporten de cierta manera que no han elegido podría socavar su valor moral, por ejemplo, al violar su autonomía o el derecho a la privacidad (López Frías, 2017). Esto es especialmente relevante en el caso de los atletas olímpicos, porque su desempeño profesional y sus vidas privadas están bajo un constante escrutinio público. La segunda limitación del ejemplarismo es que el mero hecho de que las personas consideren a los atletas como modelos a seguir no debe implicar que éstos adquieran dichas obligaciones de modo inmediato. En este sentido, Feezell (2005) apela a una de las enseñanzas más clásicas de la ética para criticar la imagen del deportista como ejemplo moral. Para ello, afirma que hemos de diferenciar entre lo que son las cosas y lo que deben ser. De no ser así, caeríamos en la conocida como «falacia naturalista», que afirma que no se pueden derivar proposiciones normativas directamente de aquello que las cosas son. El hecho de que los atletas, de hecho, sean tomados como modelos a seguir, no implica que, normativamente, deban serlo. También, siguiendo con esta crítica, la gente a menudo toma decisiones equivocadas, o actúa de modo egoísta o partidario. Por este motivo, afirmamos que la voluntad de la mayoría no debe determinar qué es correcto e incorrecto, sino que esto último debe basarse en criterios objetivos. Las obligaciones del modelo a seguir, pues, como cualquier otra obligación moral, deben estar fundamentadas en principios éticos objetivos. Tanto las sociedades, como, utilizando los 
términos de John Rawls, las «empresas cooperativas que tienen como objetivo el beneficio mutuo de todos» (Rawls, 2012) deben de fundarse en principios objetivos que todos los miembros de la sociedad pueden aceptar. La voluntad subjetiva de la mayoría no puede fundar ese tipo de obligaciones que unos sujetos se deben a otros, con el fin de sustentar la cooperación social en busca de fines comunes.

La última limitación del modelo ejemplarista tiene que ver con la posibilidad de trasladar los valores morales del deporte al ámbito de la sociedad en general. Tal y como afirma Claudio Tamburrini (2007), es dudoso que los valores del deporte influyan de algún modo en los valores que rigen el comportamiento de los individuos. Así, por ejemplo, observamos deportistas que en los terrenos de juego han de ser agresivos, pero que, sin embargo, son todo lo contrario más allá de ellos. Un caso célebre de este tipo de deportista es Ndamukong Suh, jugador de los Detroit Lions de la Liga de Fútbol Americano (NFL) que no es sólo famoso por sus labores defensivas-que requieren altas dosis de agresividad-, sino también por sus acciones filantrópicas. En 2011 fue incluido en el sexto lugar de la lista de personajes famosos que más dinero donan a la caridad, elaborada por la asociación The Giving Back Fund.

Es más, como muestra Javier Durán, no sólo es difícil mostrar empíricamente que el desarrollo moral en el deporte contribuye a otro de tipo más general, sino que «son muchas las investigaciones que han evidenciado que los practicantes deportivos evidencian menores niveles de desarrollo moral que los no practicantes» (Durán González, 2011, p. 172). Así, Törbjörn Tännsjö defiende que el atleta no debe ser un modelo a seguir. Concebirle como tal es contraproducente porque expresa un sentimiento fascista y anti-democrático que va contra los fundamentos normativos de nuestras sociedades democrático-liberales (Tännsjö, 1998). Según este autor, la admiración por los atletas de élite reproduce elementos de la ideología fascista, ya que incita a alabar a los individuos victoriosos y fuertes en detrimento de los débiles y perdedores. Esto pone en una situación difícil a aquellos que defienden el valor modélico del atleta, pues dicha admiración promueve valores que van contra aquellos a la base de nuestras sociedades, en las que no debemos distinguir entre seres superiores - vencedores - e inferiores - perdedores.

\section{Conclusión: Retos para el desarrollo de programas modelo a seguir}

En base a los enfoques filosóficos y éticos del atleta como modelo a seguir presentados en las secciones anteriores, se podría argumentar que el desarrollo de programas para convertir a los atletas en modelos a seguir se enfrenta, al menos, a los siguientes cuatro desafíos. Primero, el Movimiento Olímpico debe aclarar los tipos de comportamiento que quieren que los atletas olímpicos modelen. ¿Quieren que los atletas olímpicos sean modelos a seguir, es decir, que sirvan como ejemplos de cómo convertirse en un buen atleta? ¿O quieren que los atletas se conviertan en ejemplares morales? Si es así, ¿qué tipo de comportamientos morales quieren que sean promovidos por los atletas? ¿Son los comportamientos morales exigidos a todos y cada uno de nosotros, como el respeto por otros con diferentes creencias o características étnicas y raciales? ¿O se trata, más bien, de actos morales supererogatorios, como dar la vida para salvar a otros y donar dinero a la caridad? Además, en lo relativo a estas cuestiones, los sociólogos y los psicólogos deben participar en el debate para aclarar hasta qué punto los atletas influyen en el comportamiento atlético, moral y supererogatorio de las personas.

El segundo desafío tiene que ver con los posibles conflictos que pueden surgir entre los objetivos deportivos, las demandas morales y las acciones supererogatorias. Por ejemplo, la persecución de la victoria en una prueba olímpica podría entrar en conflicto con la personificación de los valores morales o supererogatorios, y viceversa. Un ejemplo de este tipo de conflicto es el caso de Nikki Hamblin y Abbey D'Agostino, quienes, durante la prueba femenina de los 5.000 
metros de los Juegos Olímpicos de Río en 2016, se ayudaron mutuamente a llegar a la línea de meta después de una de ellas verse envuelta en un choque accidental entre ambas. Debido al incidente, ambas corredoras no pudieron clasificarse para la carrera final. Sin embargo, dieron un ejemplo de altruismo y buen comportamiento moral que luego fue recompensado no solo con posiciones para competir en la final, sino también con el Premio del Juego Limpio de Rio 2016 (Gracious runners rewarded for fair play, 2017).

El tercer desafío surge de la necesidad de proporcionar una justificación moral para las obligaciones que los programas para convertir a los atletas en modelos a seguir exigirían a los atletas que cumplan. Este es uno de los mayores desafíos éticos a los que se enfrentan las organizaciones deportivas internacionales, ya que la práctica deportiva no tiene como objetivo principal la promoción de un carácter moral bueno, sino determinar quién sea el mejor competidor en una prueba deportiva específica. Sin embargo, Coubertin proporcionó al Movimiento Olímpico las herramientas necesarias para superar este desafío. Al concebir el Olimpismo como una «filosofía de vida» que promueve una visión específica de la buena vida y la buena sociedad, De Coubertin ubicó la protección de y obediencia a los «principios éticos fundamentales universales» (IOC, 2018), como la dignidad humana y la tolerancia, en el corazón del Olimpismo.

Dichos principios proporcionan un sólido fundamento ético para justificar obligaciones relativas a la conversión de los atletas en modelos a seguir. Su reconocimiento en el terreno del Olimpismo conduce a un cuarto desafío. El Movimiento Olímpico debe infundir en sus miembros la idea de que todas aquellas personas que representan el Movimiento están moralmente ligadas, no sólo a la obligación de evitar socavar los principios éticos fundamentales, sino también de promoverlos, contribuyendo a la búsqueda de un mundo donde los seres humanos puedan desarrollarse como personas y vivir mejor.

\section{Bibliografía}

- Amidon, S. (2012). Something like the Gods. New York: Rodale.

- Cortina, A. (2012). La leyenda del empresario excelente (1 de abril de 2012). El País.

- Cortina, A. (2014). Deporte y juego sucio (1 de febrero 2014). El País.

- Durán González, J. (2011). ¿El deporte transmite valores? En Aurora Madariaga Ortuzar y Jaime Cuenca Amigo (Eds), Los valores del ocio: cambio, choque e innovación (pp. 161-177), Bilbao: Publicaciones Universidad de Deusto, Documentos de Estudio de Ocio n. ${ }^{\circ} 43$.

- Feezell, R. (2005). Celebrated Athletes, Moral Exemplars, and Lusory Objects. Journal of the Philosophy of Sport, 32(1), 20-35. https://doi.org/10.1080/00948705.2005.9714668

- Galeano, E. (1995). El fútbol a sol y sombra, Madrid: Siglo Veintiuno de España.

- Gracious runners rewarded for fair play. (31 de mayo 2017). Accedido en 11 de marzo, 2018 en: https://www.olympic.org/news/gracious-runners-rewarded-for-fair-play

- Gomá, J. (2009) Ejemplaridad pública, Barcelona: Taurus. 
- González, E. (2012). Valdano: En la sociedad actual no hay más héroes que los deportistas, Jot Down: contemporary culture magazine. Accedido en 11 de marzo, 2018 en: https://www.jotdown.es/2012/05/jorge-valdano-en-la-sociedad-actual-no-hay-mas-heroes-quelos-deportistas/

- Jones, L. (2017). Simone Biles: The World's 100 Most Influential People. Accedido en 10 de marzo, 2018 en: http://time.com/collection/2017-time-100/4736292/simone-biles/

- IOC, (2018). Olympic Charter \& other Official IOC Documents (24 de enero 2018). Accedido en 13 de marzo, 2018 en: https://www.olympic.org/documents/olympic-charter

- López Frías, F. J. (2014). El deportista como figura moral de nuestro tiempo. Revista Internacional de Derecho y Ética del Deporte, 1, 14-28.

- López Frías, F. J. (2017). El derecho a la intimidad y los deportistas. En José Luis Pérez Triviño y Eva Cañizares Rivas (Eds.) Deporte y derechos. Madrid: Reus Editorial.

- López Frías, F. J. (2018). Football transfers and moral responsibility. Soccer \& Society, 19(4), 560-572. https://doi.org/10.1080/14660970.2016.1221822

- MacIntyre, A. C. (2013). Tras la virtud. Barcelona: Crítica.

- Malone, K. (1993). One Role Model to Another. Sports Illustrated, (14 de junio). Accedido en 9 de marzo, 2018 en: http://www.si.com/vault/1993/06/14/128740/one-role-model-to-anotherwhether-he-likes-it-or-not-charles-barkley-sets-an-example-that-many-will-follow

- Newsweek. (1993). I'm Not A Role Model. Accedido en 13 de marzo, 2018 en:http://www.newsweek.com/im-not-role-model-193808

- Píndaro (1984) Odas y Fragmentos. Madrid: Gredos.

- Rawls, J. (2012). Justicia como equidad: una reformulación. Barcelona: Paidós.

- Riggio, J. (2012). Top 25 Sports Role Models of Last 25 Years. Bleacher Report. Accedido en 10 de marzo, 2018 en: http://bleacherreport.com/articles/1043972-top-25-sports-models-of-last25-years

- Riley, K. (2017). Professional athletes serve as role models, both good and bad. Accedido en 10 de marzo, 2018 en: http://www.statepress.com/article/2017/11/spopinion-professionalathletes-serve-as-role-models-both-good-and-bad

- Spurgin, E. W. (2017). Are Coaches Obligated to Serve as Good Role Models? En R. S. Kretchmar (Ed.), Philosophy: sport (pp. 185-201). Farmington Hills, Mich.: Macmillan Reference USA.

- Tamburrini, C. (2005) Educational or Genetic Blueprints. What's the Difference? En Claudio Tamburrini y Torbjörn Tännsjö (Eds.). Genetic Technology and Sport. Ethical Questions (pp. 82-90), New York: Routledge.

- Tännsjö, T. (1998). Is Our Admiration For Sports Heroes Fascistoid? International Journal of the Philosophy of Sport, 25, 23-34.

Citius, Altius, Fortius Volumen 11 ne 2. Noviembre, 2018. ISHN. 2340-9886. http://doi.org/10.15366/eitius2018.11.2 
- The World's 100 Most Influential People. Accedido el 10 de marzo, 2018 en: http://time.com/collection/2017-time-100/

- Wellman, C. (2003). Do celebrated athletes have special responsibilities to be good role models? An imagined dialogue between Charles Barkley and Karl Malone. En J. Boxill (Ed.), Sports ethics, an anthology, (pp. 333-336). Malden, MA: Blackwell Publishing Ltd. 